\section{International Scientific Journal Theoretical \& Applied Science}

p-ISSN: 2308-4944 (print) e-ISSN: 2409-0085 (online)

Year: 2018 Issue: 09 Volume: 65

Published: $11.09 .2018 \quad$ http://T-Science.org

SECTION 7. Mechanics and machine construction.
Ravshan Indiaminov doctor of physical and mathematical sciences, professor Samarkand branch of Tashkent University of Information Technologies,

Samarkand, Uzbekistan r_indiaminov@mail.ru

Ruslan Butaev assistant Jizzakh State Pedagogical Institute, Jizzakh,Uzbekistan, samrux@umail.uz

Sobirjon Mavlanov assistant Tashkent Institute For Irrigation And Mechanization of Agricultural Engineers, Tashkent, Uzbekistan sobitoy@mail.ru

\title{
RESEARCH OF DEFORMATION OF THE CURRENT CARRYING ORTHOTROPIC SHELLS IN NONLINEAR STATEMENT
}

Abstract: In the present paper, the effect of taking into account the nonlinearity in determining the stressstrain state of flexible orthotropic shells in a geometrically nonlinear setting is studied using the example of a flexible current-carrying orthotropic conical shell located in a magnetic field.

Key words: shell, magnetic field, magneto elasticity.

Language: English

Citation: Indiaminov R, Butaev R, Mavlanov S (2018) RESEARCH OF DEFORMATION OF THE CURRENT CARRYING ORTHOTROPIC SHELLS IN NONLINEAR STATEMENT. ISJ Theoretical \& Applied Science, 09 (65): 25-30.

Soi: http://s-o-i.org/1.1/TAS-09-65-5 Doi: crossef https://dx.doi.org/10.15863/TAS.2018.09.65.5

\section{INTRODUCTION}

An important place in the mechanics of conjugate fields is occupied by the study of the motion of a continuous medium with allowance for electromagnetic effects. One of the main directions of development of modern solid mechanics is a development of the theory of conjugate fields and, in particular, the theory of the electromagnetic interaction with deformable medium $[6,7,16,17$, 20]. The electromagnetoelasticity coupled problems of anisotropic plates and shells having anisotropic conductivity are of scientific interest in terms of both theory and applications. The matter is that in the case of thin anisotropic bodies having anisotropic conductivity it is possible to solve optimal problems of magnetoelasticity by the variation of all physicalmechanical material parameters of body.

Most of the known works on the deformation of elastic conducting bodies are performed for a linearized system of equations. However, the solution of a number of applied problems, to which the nonstationary problems of determining the stressed state of flexible current-carrying anisotropic shells should be attributed, requires a more complete study of mechanical processes, including the wave fields accompanying the magnetoelastic interaction, on the basis of the nonlinear model of magnetoelasticity and represent an actual scientific problem. Problems interaction between electromagnetic field and deformed bodies are frequent in advanced technology.

\section{PROBLEM FORMULATION}

Assuming that an external magnetic field acts on the body, the magnetoelasticity equations in the Lagrangian variables in the region occupied by the body (internal problem) can be represented in the form $[1-3,5]$ :

$$
\begin{gathered}
\operatorname{rot} \vec{E}=-\frac{\partial \vec{B}}{\partial t} ; \operatorname{rot} \vec{H}=\vec{J}+\vec{J}_{c m} ; \\
\operatorname{div} \vec{B}=0, \operatorname{div} \vec{D}=0 ; \\
\rho \frac{\partial \vec{v}}{\partial t}=\rho\left(\vec{f}+\vec{f}^{\wedge}\right)+\operatorname{div} \hat{\sigma}
\end{gathered}
$$

where $\vec{E}$-electric field strength; $\vec{H}$-magnetic field strength; $\vec{B}$-magnetic induction; $\vec{D}-$ electrical induction; $\vec{J}_{\mathrm{cm}}-$ a density of foreign current , $\vec{f}$ - a volume mechanical force, $\vec{f}^{\wedge}-\mathrm{a}$ Lorentz volume force, $\vec{J}-$ a density of current, 


\begin{tabular}{|c|c|c|c|c|c|c|}
\hline Impact Factor: & $\begin{array}{l}\text { ISRA (India) } \\
\text { ISI (Dubai, UAE } \\
\text { GIF (Australia) } \\
\text { JIF }\end{array}$ & $\begin{array}{l}=1.344 \\
=0.829 \\
=0.564 \\
=1.500\end{array}$ & $\begin{array}{l}\text { SIS (USA) } \\
\text { PИНЦ (Russia) } \\
\text { ESJI (KZ) } \\
\text { SJIF (Morocco) }\end{array}$ & $\begin{array}{l}=0.912 \\
=0.156 \\
=4.102 \\
=5.667\end{array}$ & $\begin{array}{l}\text { ICV (Poland) } \\
\text { PIF (India) } \\
\text { IBI (India) }\end{array}$ & $\begin{array}{l}=6.630 \\
=1.940 \\
=4.260\end{array}$ \\
\hline
\end{tabular}

$\rho-$ is the density of the material; $\vec{v}-$ rate of deformation; $\hat{\sigma}-$ an internal stress tensor.

For the case of quadratic nonlinearity considered in $[2,3,5]$, we assume that deformations and shifts are small in comparison with the angles of rotation of the element, and the angles themselves are substantially less than unity. Elastic properties of the shell are considered orthotropic, which main directions of elasticity coincide with the directions of the corresponding coordinate lines. Material obeys the generalized Hooke's law and has a finite conductivity. Electromagnetic properties of the material of the current-carrying shell are characterized by tensors of electrical conductivity $\sigma_{i j}$, magnetic permeability $\mu_{i j}$ and dielectric permittivity $\varepsilon_{i j}(i, j=1,2,3)$. At the same time due to the crystallophysics for the considered class of conducting media with rhombic crystal structure it was considered that the tensors $\sigma_{i j}, \mu_{i j}, \varepsilon_{i j}$ take a diagonal form $[4,11,19]$.

System of equations must be closed magnetoelasticity relations linking the vectors of the electromagnetic field and induction, as well as Ohm's law defining the conduction current density in a movable medium. If the body is linear with respect to the anisotropic magnetic and electrical properties, the constitutive equations for the electromagnetic field characteristics and kinematic equations for the electrical conductivity, as well as expressions for the Lorentz forces, taking into account the external current $\vec{J}_{c m}$ into the Lagrangian variables are written respectively as:

$$
\begin{gathered}
\vec{B}=\mu_{i j} \vec{H}, \vec{D}=\varepsilon_{i j} \vec{E}, \\
\vec{J}=\sigma_{i j} \Gamma F^{T} F^{-1}\left[\vec{J}{ }_{c m}+\vec{E}+\vec{v} \times \vec{B}\right], \\
\rho \vec{f}^{\wedge}=\Gamma^{-1} F^{-1}\left[\vec{J}_{c m} \times \vec{B}+\sigma_{i j}(\vec{E}+\vec{v} \times \vec{B}) \times \vec{B}\right]
\end{gathered}
$$

Here $\sigma_{i j}, \varepsilon_{i j}, \mu_{i j}$ are the tensors of electrical conductivity, dielectric and magnetic permittivities of linear current-carrying anisotropic body $(i, j=1,2,3) \quad$ respectively. For homogeneous anisotropic media, they are symmetric second-rank tensors. Thus, equations (1) and (2) together with (3)-(5) are a closed system of nonlinear equations of magnetoelasticity for anisotropic current-carrying bodies with anisotropic electrical conductivity, magnetic and dielectric permittivities in the Lagrangian formulation.

Coordinate surface in the unstrained state we assign to the curvilinear orthogonal coordinate system $S$ and $\theta$, where $S$ - length of the arc forming (meridian) is measured from a fixed point, $\theta$ - central angle in a parallel circle measured from the selected plane. The coordinate lines $s=$ const and $\theta=$ const lines are the principal curvatures of the surface coordinate. Choosing a coordinate $\zeta$ coordinate normal to the surface of revolution, we refer to the shell of the spatial coordinate system of coordinates $s, \theta, \zeta$. Assume that the surface of the conical shell known magnetic induction, and the surface mechanical strength. Upon receipt of the resolution of the system in the normal form of Cauchy choose as basic functions $u, w, \theta_{S}, N_{S}, Q_{S}, M_{S}, B_{\zeta}, E_{\theta}$.

By selecting these functions in the future, you can choose different combinations of fixing cone. We assume that all the components of the excited electromagnetic field and displacement field belonging to magneto-elasticity problem equation does not depend on the coordinates $\theta$, and also believe that the elastic characteristics and electromagneto-mechanical shell material does not vary along the parallels.

After some transformations [11], we obtain a complete system of nonlinear differential equations in the form magnetoelasticity Cauchy, which describes the stress-strain state of the currentcarrying orthotropic conical shell with an unsteady mechanical and magnetic fields:

$$
\begin{gathered}
\frac{\partial u}{\partial s}=\frac{1-v_{s} v_{\theta}}{e_{s} h} N_{S}-\frac{v_{\theta} \cos \varphi}{r} u- \\
-\frac{v_{\theta} \sin \varphi}{r} w-\frac{1}{2} \theta_{S}^{2} ; \frac{\partial w}{\partial s}=-\theta_{S} \\
\frac{\partial \theta_{S}}{\partial s}=\frac{12\left(1-v_{s} \nu_{\theta}\right)}{e_{s} h^{3}} M_{S}- \\
-\frac{\nu_{\theta} \cos \varphi}{r} \theta_{S} ; \\
\frac{\partial N_{S}}{\partial s}=\frac{\cos \varphi}{r}\left(\left(v_{S} \frac{e_{\theta}}{e_{S}}-1\right) N_{S}+\right. \\
\left.+e_{\theta} h\left(\frac{\cos \varphi}{r} u+\frac{\sin \varphi}{r} w\right)\right)- \\
-P_{S}+h J_{\theta C T} B_{\zeta}-\sigma_{1} h\left[E_{\theta} B_{\zeta}+\right. \\
\left.0.5 \frac{\partial w}{\partial t} B_{\zeta}\left(B_{S}^{+}+B_{S}^{-}\right)-\frac{\partial u}{\partial t} B_{\zeta}^{2}\right]+\rho h \frac{\partial^{2} u}{\partial t^{2}} \\
\frac{\partial Q_{S}}{\partial s}=-\frac{\cos \varphi}{r} Q_{S}+v_{S} \frac{e_{\theta}}{e_{S}} \frac{\sin \varphi}{r} N_{S}+ \\
+e_{\theta} h \frac{\sin \varphi}{r}\left(\frac{\cos \varphi}{r} u+\frac{\sin \varphi}{r} w\right)-P_{\zeta}- \\
-0.5 h J_{\theta C T}\left(B_{S}^{+}+B_{S}^{-}\right)-\sigma_{3} h\left[-0.5 E_{\theta}\left(B_{S}^{+}+B_{S}^{-}\right)-\right. \\
-0.25 \frac{\partial w}{\partial t}\left(B_{S}^{+}+B_{S}^{-}\right)^{2}-
\end{gathered}
$$




$$
\begin{gathered}
-\frac{1}{12} \frac{\partial w}{\partial t}\left(B_{S}^{+}-B_{S}^{-}\right)^{2}+0.5 \frac{\partial u}{\partial t} B_{\zeta}\left(B_{S}^{+}+B_{S}^{-}\right)+ \\
\left.+\frac{h}{12} \frac{\partial \theta_{S}}{\partial t} B_{\zeta}\left(B_{S}^{+}+B_{S}^{-}\right)\right]+\rho h \frac{\partial^{2} w}{\partial t^{2}} \\
\frac{\partial M_{S}}{\partial s}=\frac{\cos \varphi}{r}\left[\left(v_{S} \frac{e_{\theta}}{e_{S}}-1\right) M_{S}+\right. \\
\left.+\frac{e_{\theta} h^{3}}{12} \frac{\cos \varphi}{r} \theta_{S}\right]+Q_{S}+N_{S} \theta_{S}- \\
-\frac{\sin \varphi}{r}\left(v_{S} \frac{e_{\theta}}{e_{S}} M_{S}+\frac{e_{\theta} h^{3}}{12} \frac{\cos \varphi}{r} \theta_{S}\right) \theta_{S}+ \\
+\frac{h^{3}}{12} \frac{\partial^{2} \theta_{S}}{\partial t^{2}} ; \\
\frac{\partial B_{\zeta}}{\partial s}=-\sigma_{2} \mu\left[E_{\theta}+0.5 \frac{\partial w}{\partial t}\left(B_{S}^{+}+B_{S}^{-}\right)-\frac{\partial u}{\partial t} B_{\zeta}\right]+ \\
+\frac{B_{S}^{+}-B_{S}^{-}}{h} ; \\
\frac{\partial E_{\theta}}{\partial s}=-\frac{\partial B_{\zeta}}{\partial t}-\frac{\cos \varphi}{r} E_{\theta} .
\end{gathered}
$$$$
\text { Here } N_{s}, \quad N_{\theta}-\text { meridional and }
$$
circumferential forces; $S$-shear; $Q_{s},-$ shear force; $M_{s}, M_{\theta}-\quad$ bending moments; $u, w-$ displacement and deflection; $\theta_{S}$-the rotation angle of the normal; $P_{S}, P_{\zeta}-$ mechanical load components; $E_{\theta}$ - mechanical load components; $B_{\zeta}$ - the normal component of the magnetic induction; $B_{s}^{+}, B_{s}^{-}-$known components of the magnetic induction on the surface of the shell; $J_{\theta \mathrm{cm}}{ }^{-}$ component of the electric current density from an external source; $e_{s}, e_{\theta}$ - elastic modules in the directions $s, \theta-$ respectively; $v_{S}, v_{\theta}-$ Poisson's ratio, which characterize the tensile transverse compression in the direction of the coordinate axes; $\mu-$ permeability; $\omega-$ the angular frequency; $\sigma_{1}, \sigma_{2}, \sigma_{3}$ - the main components of the tensor conductivity.

Obtained coupled allowing system of nonlinear differential equations of order eight (6) describes the stress-strain state of flexible current-carrying orthotropic conical shells of rotation having orthotropic electrical conductivity, magnetic and electrical permittivity. Solving of magnetoelasticity boundary values problems associated with the essential computational difficulties.

This is because the resolution of the system of equations (6) is a system of differential equations of hyperbolic-parabolic type of eighth-order with variable coefficients. Components of the Lorentz force consider the speed of shell deformation, an external magnetic field, the size and intensity of the conduction current relatively to the external magnetic field. Accounting for nonlinearity in the equations of motion causes nonlinearity in the ponderomotive force. The boundary conditions for the functions characterizing the mechanical part of the problem are as in the usual theory of shells. The boundary conditions for electromagnetic functions can be specified taking into account the electric field or a combination of electrical and magnetic fields. The initial conditions are given in the classical form.

\section{METHODOLOGY OF THE SOLUTION}

The method for solving the nonlinear problem of the magnetoelasticity of shells is based on the consistent use of the Newmark scheme, the quasilinearization method, and the discrete orthogonalization method $[3,8-15,18]$. To separate the variables from the time coordinate, we apply the implicit Newmark scheme, with which the nonlinear boundary value problem reduces to a sequence of nonlinear one-dimensional boundary value problems at each time step. The next step in solving the sequence of nonlinear boundary value problems of magnetoelasticity is based on the application of the quasilinearization method, with which the nonlinear boundary value problem reduces to a sequence of linear boundary value problems. Then each of the linear boundary value problems of the sequence on the corresponding time interval is solved numerically with the help of the stable method of discrete orthogonalization.

\section{THE DISCUSSION OF THE RESULTS}

A study of the stress-strain state of flexible orthotropic conical shell of boroaluminum of constant thickness $h=5 \cdot 10^{-4} \mathrm{~m}$, under mechanical stress $P_{\zeta}=5 \cdot 10^{3} \sin \omega t \mathrm{~N} / \mathrm{m}^{2}$ was performed. The shell is in an external magnetic field $B_{S 0}=0.1 T$ and is applied by the external electrical current of $J_{\theta C T}=-5 \cdot 10^{4} \sin \omega t \mathrm{~A} / \mathrm{m}^{2}$, density. The shell has a finite orthotropic conductivity $\sigma\left(\sigma_{1}, \sigma_{2}, \sigma_{3}\right)$. Note that in this case the anisotropy of specific electrical resistivity is $\eta_{3} / \eta_{1}=2.27$.

We assume that by the electric current in the disturbed state is evenly distributed on the shell, the external current density does not depend on the coordinates. In this case, the combined effect on the shell loading, the ponderomotive force consisting of Lorentz forces and mechanical.

The boundary conditions are $s=s_{0}=0: u=0, Q_{S}=-200, M_{S}=0, B_{\zeta}=0.3 \sin \omega t T ;$ $s=s_{N}=0.4 m: w=0, \quad \theta_{S}=0, \quad B_{\zeta}=0$. 


\begin{tabular}{|c|c|c|c|c|c|c|}
\hline Impact Factor: & $\begin{array}{l}\text { ISRA (India) } \\
\text { ISI (Dubai, UAE } \\
\text { GIF (Australia) } \\
\text { JIF }\end{array}$ & $\begin{array}{l}=1.344 \\
=0.829 \\
=0.564 \\
=1.500\end{array}$ & $\begin{array}{l}\text { SIS (USA) } \\
\text { PИНЦ (Russia) } \\
\text { ESJI (KZ) } \\
\text { SJIF (Morocco) }\end{array}$ & $\begin{array}{l}=0.912 \\
=0.156 \\
=4.102 \\
=5.667\end{array}$ & $\begin{array}{l}\text { ICV (Poland) } \\
\text { PIF (India) } \\
\text { IBI (India) }\end{array}$ & $\begin{array}{l}=6.630 \\
=1.940 \\
=4.260\end{array}$ \\
\hline
\end{tabular}

The initial conditions are

$$
\begin{gathered}
\left.\vec{N}(s, t)\right|_{t=0}=0,\left.\quad \dot{u}(s, t)\right|_{t=0}=0,\left.\quad \dot{w}(s, t)\right|_{t=0}=0 \\
\text { The parameters of the shell and the material are: } \\
s_{0}=0, s_{N}=0,4 m, h=5 \cdot 10^{-4} \mathrm{~m}, \quad r_{0}=0.4 \mathrm{~m} \\
r=r_{0}+s \cos \varphi ;, \omega=314.16 \mathrm{sec}^{-1}, \varphi=\pi / 15, \\
\rho=2300 \mathrm{~kg} / \mathrm{m}^{3}, B_{s}^{+}=B_{s}^{-}=0.5 \mathrm{~T} ., \\
\mu=1.256 \cdot 10^{-6} \mathrm{H} / \mathrm{m}, J_{\theta c m}=-5 \cdot 10^{5} \sin \omega t \mathrm{~A} / \mathrm{m}^{2}, \\
\sigma_{1}=0.454 \cdot 10^{8}(\Omega \times m)^{-1}, \sigma_{2}=0.454 \cdot 10^{8}(\Omega \times m)^{-1}, \\
\sigma_{3}=0.200 \cdot 10^{8}(\Omega \times m)^{-1}, v_{S}=0.262, v_{\theta}=0.320, \\
P_{\zeta}=5 \cdot 10^{3} \sin \omega t \mathrm{~N} / \mathrm{m}^{2}, B_{S 0}=0.1 T \\
e_{S}=22.9 \cdot 10^{10} \mathrm{~N} / \mathrm{m}^{2}, e_{\theta}=10.7 \cdot 10^{10} \mathrm{~N} / \mathrm{m}^{2}
\end{gathered}
$$

The solution is found in the time interval $\tau=0 \div 10^{-2} \mathrm{sec}$ for the integration time step is chosen to be $\Delta t=1 \cdot 10^{-3} \mathrm{sec}$. Maximum values obtained at time step $t=5 \cdot 10^{-3} \mathrm{sec}$.

It was investigated the stress-strain states of flexible shells in nonlinear formulation based on comparison of the solutions obtained for the currentcarrying orthotropic cone of beryllium and currentcarrying isotropic cone of aluminum, as well as for the isotropic cone of aluminum in the absence of a magnetic field and the external current.

In Fig. 1 shows the deflection distribution according to "s" at $t=5 \cdot 10^{-3} \mathrm{sec}$. The results of the calculations are given for the variants: 1 - a currentcarrying isotropic cone made of aluminum; 2 is an isotropic cone made of aluminum in the absence of a magnetic field and an external current; 3 - currentcarrying orthotropic cone of beryllium. In all three cases the distribution of the deflection is nonlinear ( $\left.1 \leq w / h_{0} \leq 5\right)$ and its maximum values occur in the left contour of the shell. Thus, in the case of beryllium orthotropic cone and the cone of isotropic alumina, considering the maximum value of the magnetic field deflection differs by about two times.

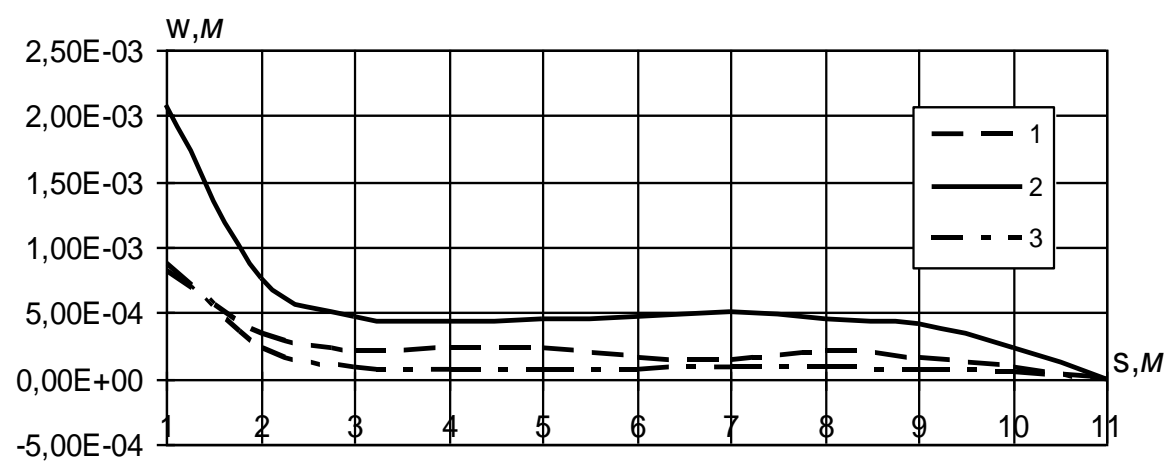

Figure 1 - Distribution deflection $w(s)$ at $t=5 \cdot 10^{-3} \mathrm{sec}$ for all variants.

It was revealed that in the case of the isotropic cone without influence of the magnetic and electric fields the deflection increases significantly ( $\left.w / h_{0}=4\right)$. This is because in the absence of an electric field acting on the shell the tensile strength of the tangential component of the magnetic induction ( $B_{s}^{ \pm}$) and the tangential component of the Lorentz force $\left(\rho F_{\mathrm{S}}^{\wedge}\right)$ is equals to zero. In this case the shell becomes more ductile, i.e. flexible with respect to the deflectio n. The absence of a magnetic field ( $\left.B_{\zeta}=0\right)$ also leads to an increase in deflection.
Figures 2 and 3 show the distributions of the maximum stresses $\sigma_{22}^{+}(s)+T_{22}^{+}(s)$ and meridional bending moment $M_{S}$ along the meridian of the shell at the time point $t=5 \cdot 10^{-3} \mathrm{sec}$ for all variants: 1 - the current-carrying orthotropic cone of beryllium; 2 - current-carrying isotropic cone made of aluminum; 3 is an isotropic cone made of aluminum in the absence of a magnetic field and an external current.

$$
\sigma_{22}^{+}+T_{22}^{+}, N / m^{2}
$$




\begin{tabular}{|c|c|c|c|c|c|c|}
\hline Impact Factor: & $\begin{array}{l}\text { ISRA (India) } \\
\text { ISI (Dubai, UAE } \\
\text { GIF (Australia) } \\
\text { JIF }\end{array}$ & $\begin{array}{r}=1.344 \\
=0.829 \\
=0.564 \\
=1.500\end{array}$ & $\begin{array}{l}\text { SIS (USA) } \\
\text { PИНЦ (Russia) } \\
\text { ESJI (KZ) } \\
\text { SJIF (Morocco) }\end{array}$ & $\begin{array}{l}=0.912 \\
=0.156 \\
=4.102 \\
=5.667\end{array}$ & $\begin{array}{l}\text { ICV (Poland) } \\
\text { PIF (India) } \\
\text { IBI (India) }\end{array}$ & $\begin{array}{l}=6.630 \\
=1.940 \\
=4.260\end{array}$ \\
\hline
\end{tabular}

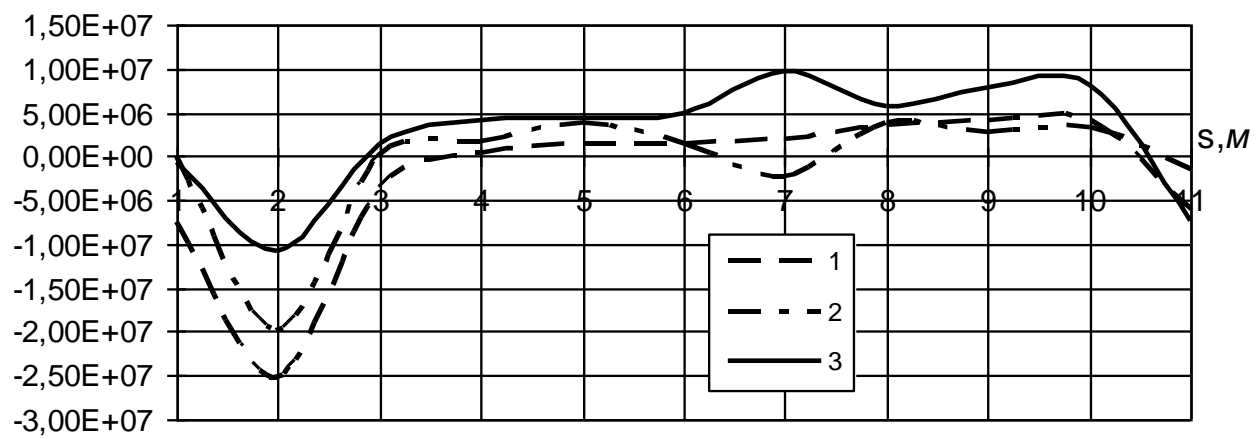

Figure 2 - Distributions of the maximum stresses $\sigma_{22}^{+}+T_{22}^{+}$at $t=5 \cdot 10^{-3} \mathrm{sec}$ for all variants. $M_{S}, N \cdot m / m$

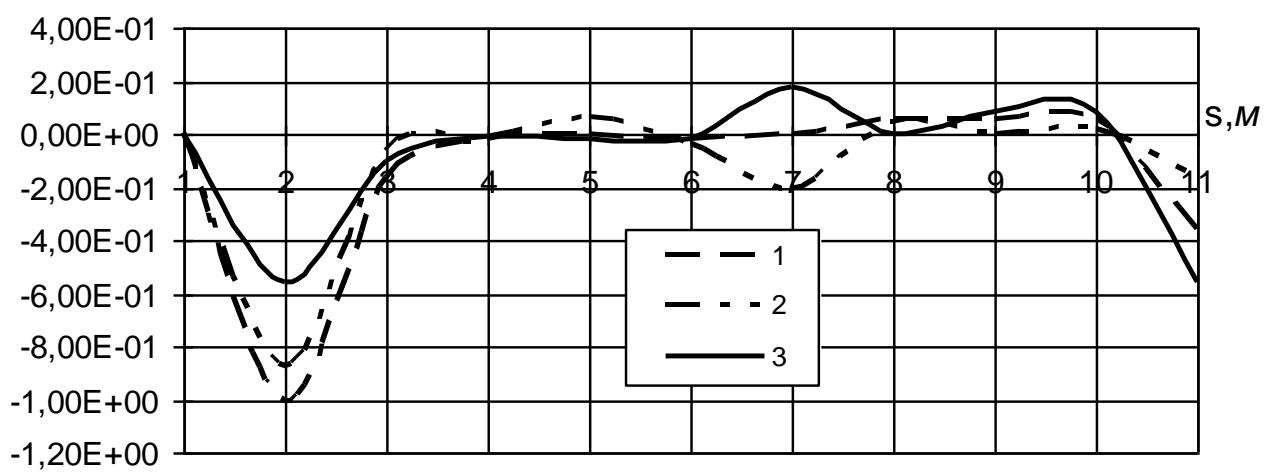

Figure 3 - Distribution meridional bending moment $M(s)$ at $t=5 \cdot 10^{-3} \mathrm{sec}$ for all variants for all variants.

Note that when solving the problem, the stress of the conical shell was considered as the sum of Maxwell's mechanical stresses and stresses, i.e. the total stress state was taken into account. It can be seen from these curves that the distribution of the change in stresses and bending moment differs quantitatively and qualitatively. A phase-independent distribution of stresses and bending moment is observed on a segment $0.08 \mathrm{~m}<\mathrm{s}<0.4 \mathrm{~m}$. Their maximum values arise near the left-hand section of the shell at $s=0.04 m$. In this case, in the presence of a magnetic field and an external current and their absence, the values of stresses and bending moment in differ by 1.5 times. The obtained results show the influence of orthotropic electrical conductivity, external electric current and external magnetic field on the stress-strain state of the shell, and the inclusion of geometric nonlinearity makes it possible to substantially clarify the deformation picture.

\section{CONCLUSION}

The nonlinear problem of magnetoelasticity is considered in the axisymmetric statement for conical shells. The resolving system of nonlinear differential equations is obtained, which describes the stressstrain state of the flexible orthotropic conical shell in mechanical and magnetic fields. The numerical example is given. The stress-strain state of flexible shells in nonlinear formulation based on comparison of the solutions obtained for the current-carrying orthotropic cone of beryllium and current-carrying isotropic cone of aluminum, as well as for the isotropic cone of aluminum in the absence of a magnetic field and the external current was investigated. In all three cases the distribution of the non-linear deflection and its maximum values occur in the left contour of the shell. At the same time in the case of beryllium orthotropic cone and the isotropic cone of aluminum, considering the magnetic field maximum values of deflection differ by about two times. It was revealed that in the case of the isotropic cone without influence of the magnetic and electric fields, the deflection increases significantly. This is because in the absence of an electric field acting on the shell, the tensile strength of the tangential component of the magnetic induction, and the tangential component of the Lorentz force equals to zero. 


\begin{tabular}{l|lr|ll|ll} 
& ISRA (India) & $=\mathbf{1 . 3 4 4}$ & SIS (USA) & $=\mathbf{0 . 9 1 2}$ & ICV (Poland) & $=\mathbf{6 . 6 3 0}$ \\
Impact Factor: & ISI (Dubai, UAE) $=\mathbf{0 . 8 2 9}$ & PUHЦ (Russia) $=\mathbf{0 . 1 5 6}$ & PIF (India) & $=\mathbf{1 . 9 4 0}$ \\
& GIF (Australia) & $=\mathbf{0 . 5 6 4}$ & ESJI (KZ) & $=\mathbf{4 . 1 0 2}$ & IBI (India) & $=\mathbf{4 . 2 6 0}$ \\
& JIF & $=\mathbf{1 . 5 0 0}$ & SJIF (Morocco) & $\mathbf{5 . 6 6 7}$ & & \\
\hline
\end{tabular}

\section{References:}

1. A. Ambartsumyan, G.E. Bagdasaryan, and M.V. Belubekyan, (1977) Magnetoelasticity of Thin Shells and Plates [in Russian], Nauka, Moscow.

2. Ya. M. Grigorenko and L. V. Mol'chenko, (1993) Fundamentals of the Theory of Plates and Shells [in Ukrainian], Lybid', Kyiv.

3. Ya. M. Grigorenko and L. V. Mol'chenko, (2010) Fundamentals of the Theory of Plates and Shells with Elements of Magnetoelasticity (Textbook) [in Russian], IPTs Kievskii Universitet, Kyiv.

4. Yu. I. Sirotin and M.P. Shaskol'skaya, (1979) Fundamentals of Crystal Physics [in Russian], Nauka, Moscow.

5. A. F. Ulitko, L. V. Mol'chenko, and V. F. Koval'chuk, (1994) Magnetoelasticity under Dynamic Loading [in Ukrainian], Lybid', Kyiv.

6. H. G. Lippmann, (1976) "Principle de la conservation de l'electricite," Ann. Chim., No. $2,17-35$.

7. G.A. Maugin, (1985) Nonlinear Electromechanical Effects and Applications, World Scientific, Singapore.

8. L. V. Mol'chenko and I. I. Loos, (2010) "Influence of conicity on the stress-strain state of a flexible orthotropic conical shell in a nonstationary magnetic field," Int. Appl. Mech., 46, No. 11, 1261-1267. MathSciNetCrossRefMATH

9. L. V. Mol'chenko, I. I. Loos, and L. M. Fedorchenko, (2013) "Axisymmetric magnetoelastic deformation of a flexible orthotropic ring with orthotropic conductivity," Int. Appl. Mech., 49, No. 3, 322327. ADSMathSciNetCrossRefMATH

10. L. V. Mol'chenko, I. I. Loos, and R. Sh. Indiaminov, (1997) "Nonlinear deformation of conical shells in magnetic fields," Int. Appl. Mech., 33, No. 3, 221-226.

11. L. V. Mol'chenko, I. I. Loos, and R. Sh. Indiaminov, (2008) "Determining the stress state of flexible orthotropic shells of revolution in magnetic field," Int. Appl. Mech., 44, No. 8, 882-891. ADSMathSciNetCrossRefMATH

12. L. V. Mol'chenko, I. I. Loos, and R. Sh. Indiaminov, (2009) "Stress-strain state of flexible ring plates of variable stiffness in a magnetic field," Int. Appl. Mech., 45, No. 11, 1236-1242. ADSMathSciNetCrossRefMATH

13. Indiaminov R.SH. (2015) Magnetoelastic deformation of a current-carrying orthotropic conical shell with an ortotropiya of the carrying-out properties // Bulletin of the University of Kiev. Series: Physics \& Mathematics. No.5, $81-86$.

14. R. Sh.Indiaminov., U.Akbaev., A.Dustmuradov. (2016) Nonlinear deformation of flexible orthotropic shells of variable thickness in the non stationary magnetic field // International Journal of Engineering Innovation \& Research., - Volume 5, Issue 3, 234-238.

15. R.Indiaminov., A.Hotamov., F.Akhmedov. (2017) Magnetoelastic deformation of the current carrying shells with the orthotropy of conductive properties // International Journal of Engineering Innovation \& Research., Volume 5, Issue 6, 344-350.

16. F. C. Moon, (1984) Magneto-Solid Mechanics, John Wiley \& Sons, New York.

17. F. C. Moon and S. Chattopadhyay, (1974) "Magnetically induced stress waves in a conducting solid: Theory and experiment," Trans. ASME, J. Appl. Mech., 41, No. 3, 641-646.

18. N. M. Newmark, (1959) "A method of computation for structural dynamics," ASCE, J. Eng. Mech. Div., 85, No. 7, 67-97.

19. J. F. Nye, (1964) Physical Properties of Crystals, Clarendon Press, Oxford. MATH

20. Y.-H. Pao and K. Hutter, (1975) "Electrodynamis for moving elastic solids and viscous fluids," Proc. IEEE, 63, No. 7, 10111021. MathSciNetCrossRef 